\title{
Efectividad del vendaje neuromuscular propioceptivo en pacientes con dolor cervical mecánico crónico en un hospital estatal, Lima, Perú
}

Violeta Del Aguila Torres ${ }^{1}$; Janelle Lermo Fajardo ${ }^{\text {; }}$ Cristhian Santiago Bazán*

\section{RESUMEN}

Objetivo: Determinar la influencia de la aplicación del vendaje neuromuscular propioceptivo (VNP) en pacientes con dolor cervical mecánico crónico (DCMC) en un hospital estatal, Lima, Perú.

Materiales y métodos: Estudio de enfoque cuantitativo, de tipo cuasi experimental y de corte longitudinal, prospectivo realizado entre abril y junio del 2018. La muestra estuvo formada por 30 pacientes, divididos en dos grupos de 15 participantes cada uno: el grupo experimental (en que se aplicó el VNP) y un grupo control (que empleó el tratamiento convencional). En ambos grupos se consideró una evaluación de entrada y una evaluación de salida. Para evaluar el dolor se utilizó la escala análoga del dolor y para medir el grado de discapacidad se empleó el cuestionario de índice de discapacidad cervical. Para el análisis estadístico se utilizó el programa Stata v13.0 (con la prueba t Student) que confirma la diferencia significativa que existe entre los promedios del pretest y el postest.

Resultados: La aplicación del VNP influye estadísticamente en la disminución del dolor cervical mecánico crónico $(p=0,000)$ y una mejora del índice de discapacidad cervical $(p=0,000)$.

Conclusiones: Se demostró una influencia estadísticamente significativa en la disminución del dolor cervical y el índice de discapacidad en pacientes con DCMC. Por lo tanto, este procedimiento se debería considerar como parte del tratamiento de DCMC.

Palabras clave: Dolor de cuello; Fisioterapia; Modalidades de fisioterapia (Fuente: DeCS BIREME).

\section{Effectiveness of the proprioceptive neuromuscular taping in patients with chronic mechanical neck pain at a state hospital in Lima, Peru}

\section{ABSTRACT}

Objective: To determine the influence of applying a proprioceptive neuromuscular taping (VNP) on patients with chronic mechanical neck pain (DCMC) at a state hospital in Lima, Peru.

Materials and methods: A quantitative-approach, quasi-experimental, longitudinal, prospective study conducted between April and June 2018. The sample consisted of 30 patients divided into two groups of 15 participants each: an experimental group (in whom the VNP was applied) and a control group (in whom the conventional treatment was applied). Both groups underwent a pretest and a posttest. Pain was evaluated by the analogue pain scale and the degree of disability was measured by the neck disability index questionnaire. The statistical analysis was performed using the Stata 13 program and the Student's t-test, which confirms that there is a significant difference between the pretest and posttest averages. Results: The application of the VNP statistically influences the reduction of the chronic mechanical neck pain $(p=0.000)$ and the improvement of the neck disability index $(p=0.000)$.

Conclusions: A statistically significant influence was shown on the reduction of neck pain and the disability index in patients with DCMC. Therefore, this medical procedure should be taken into consideration as part of the DCMC treatment.

Keywords: Neck pain; Physical therapy; Physical therapy modalities (Source: MeSH NLM).

1 Universidad Católica Sede Sapientiae. Lima, Perú.

2 Centro de Rehabilitación Integral Física Funcional. Lima, Perú.

*Autor corresponsal. 


\section{INTRODUCCIÓN}

El dolor cervical crónico (DCMC) conlleva una gran variedad de signos y síntomas como la tensión neural y las alteraciones conductuales. Este tipo de lesión es frecuente en la práctica médica y su recuperación presenta un alto costo para los pacientes ${ }^{(1-3)}$.

Este padecimiento tiene un impacto en el estado funcional del paciente, interfiere en las actividades básicas como el sueño, el descanso o ambas, el cuidado personal y las actividades laborales. Si consideramos que la zona cervical es una región de alto riesgo, el DCMC puede convertirse en una causa común de discapacidad y absentismo laboral (4).

En los pacientes con DCMC, generalmente se observa rigidez en los grupos musculares del cuello como en la porción superior del trapecio, las escápulas elevadoras y los músculos esternocleidomastoideos (ECOM), lo que ocasiona la limitación del movimiento ${ }^{(5)}$, así como una disminución de la fuerza de los músculos flexores y extensores cervicales ${ }^{(6)}$.

La cervicalgia tiene, fundamentalmente, un origen mecánico, es ocasionada por posturas mantenidas de cabeza, brazos, o ambos, por sostener o cargar pesos de manera estática, realizar movimientos repetitivos de la columna cervical o de los miembros superiores, así como no realizar pausas o descansos en la actividad laboral del individuo. Si la lesión es constante y repetida podría dañar los discos intervertebrales y hasta las vértebras, lo que ocasiona una lesión nerviosa, enfermedades reumáticas y traumatismos que dan lugar a irritación de los nervios ${ }^{(7,8)}$.

Existen factores que predisponen al dolor de cuello como el uso de la computadora ${ }^{\left({ }^{9}\right)}$, uso de mensajería móvil ${ }^{(10)}$, carga laboral (11), posturas inadecuadas, cambios degenerativos, lesiones y otras afecciones que generan, en forma directa o indirecta, episodios de dolor cervical (12), acompañados de una limitación de la movilidad y funcionalidad del cuello. Por lo expuesto, estos factores pueden tener un impacto negativo en la calidad de vida relacionada con la salud.

En la actualidad existen diversos tipos de abordaje para el tratamiento del DCMC, entre ellos, la intervención de la aplicación de la terapia física y rehabilitación, que es un medio eficaz en el tratamiento para el tratamiento de este cuadro ${ }^{(13)}$.

El VPN es una técnica utilizada en fisioterapia y rehabilitación creada en 1979 por el Dr. Kenzo Kase. Consiste en un vendaje elástico autoadhesivo que permite la recuperación de la parte lesionada sin disminuir su función corporal y que se ha empleado en el manejo de muchas alteraciones del sistema musculoesquelético ${ }^{(14)}$. El VNP es una herramienta complementaria en la intervención terapéutica tanto en deportistas como en pacientes que padecen algún tipo de lesión musculoesquelética; su aplicación permite un menor riesgo para el paciente y su pronta recuperación con el menor número de secuelas ${ }^{(15)}$.

\section{MATERIALES Y MÉTODOS}

\section{Diseño y población de estudio}

La investigación tiene un enfoque cuantitativo. Es un estudio cuasiexperimental con diseño de tipo longitudinal. La muestra fue de 30 pacientes diagnosticados con DCMC, que se organizaron en dos grupos de estudio: el grupo control $(n=15)$ y el grupo experimental $(n=15)$. Los criterios de inclusión fueron presentar dolor cervical mecánico, aceptar participar libremente en el estudio, previa firma del consentimiento informado, no haber tenido alguna intervención quirúrgica a nivel de la región cervical, algún tipo de traumatismo, enfermedad degenerativa, o ambos.

Entre los criterios de exclusión estuvo no otorgar el consentimiento informado, haber tenido una intervención quirúrgica a nivel cervical, padecer artrosis cervical, degeneración osteoarticular, o ambas; presentar algún tipo de problema genético, congénito, o ambos; se excluyó también a los pacientes que hayan sufrido un traumatismo (síndrome de latigazo, fractura cervical) o algún dolor por cuestiones neurológicas (polineuropatía diabética, ACV). En el grupo control se aplicó la terapia convencional y en el grupo experimental se empleó el VNP.

\section{Variables y mediciones}

Las variables utilizadas en el estudio fueron el dolor cervical que se midió con la escala visual análoga del dolor (EVA), y la discapacidad cervical que se calculó con el índice de discapacidad cervical (IDC). Ambas variables se midieron en los grupos en una evaluación inicial (pretest) y una evaluación final (postest).

Para la obtención de la muestra se empleó la técnica de muestreo no probabilístico o criterio de los autores. Así mismo, las informaciones sociodemográfica y clínica se registraron en una ficha de recolección de datos.

El procedimiento en el grupo control (convencional) consistió en la aplicación de la compresa húmeda caliente (CHC), así como masoterapia, y también el estiramiento de la zona cervical.

El procedimiento se realizó en dos fases. En la primera a ambos grupos se les solicitó el consentimiento informado y se les explicó el procedimiento a seguir.

En el grupo experimental, en la sesión inicial de la segunda fase, se evaluó el dolor con EVA y la funcionabilidad del paciente con el IDC. De la segunda a la última sesión 
se aplicó el VNP de color azul, lo que generó un efecto relajante y la disminución de la temperatura. El vendaje permaneció adherido por 5 semanas y se retiró. Luego se midieron, nuevamente, el dolor y la funcionabilidad con las mismas escalas. El vendaje neuromuscular propioceptivo fue colocado en dos músculos principales (fibras superiores del ECOM) con dirección de inserción a origen en forma "Y" (lo que genera un efecto de relajación muscular), que está indicado para zonas pequeñas o lineales, y se usa por encima del vientre muscular, sin sacarlo. Por otro lado, para una adecuada aplicación del VNP la piel fue limpiada con alcohol y rasurada, si era necesario.

En el grupo control, durante la segunda fase, se empleó el tratamiento convencional usado en fisioterapia y rehabilitación, que consistió en la aplicación de la compresa húmeda caliente $(\mathrm{CHC})$, masoterapia y el estiramiento de la zona cervical. Por último, en la última sesión se miden nuevamente las variables ya expuestas.

\section{Análisis estadístico}

Para el procesamiento de la información de los datos obtenidos se empleó el programa estadístico Stata v13.0. Se utilizó la estadística inferencial, y se comprobaron si los resultados postintervención son significativos frente a los datos obtenidos en la preintervención. Se aplicó la prueba de normalidad basada en la prueba de Shapiro-
Wilk. Así mismo, se usó la prueba paramétrica de t Student para muestras relacionadas, con un nivel de significancia del $5 \%$.

\section{Consideraciones éticas}

Para realizar la investigación se tuvieron presentes las consideraciones bioéticas en la investigación científica (justicia, autonomía, beneficencia y no maleficencia). Además, antes de recolectar la información, se explicó a los participantes el procedimiento, la técnica aplicada en el estudio, los beneficios y riesgos de la investigación. Se garantizó el respeto a la privacidad y a la libre participación. Por otro lado, no hubo discriminación en el grupo de participantes.

\section{RESULTADOS}

En la tabla 1 se observa que, al aplicar el vendaje neuromuscular propioceptivo (VNP), existe una mejora crónica, de acuerdo a la prueba paramétrica T Student para muestras independientes que muestra una diferencia estadísticamente significativa $(p=0,000)$. Así mismo, en el grupo experimental, los pacientes obtuvieron mejores resultados en sus puntajes: antes de la aplicación del VNP $(7,73 \pm 2,12)$, y después de la aplicación del VNP $(6,13 \pm 1,12)$.

Tabla 1. Efectividad del vendaje neuromuscular propioceptivo y mejoría del dolor cervical crónico en pacientes de un hospital estatal. Lima, Perú

\begin{tabular}{|c|c|c|c|}
\hline Estadístico & Grupo control & Grupo experimental & t Student \\
\hline & $\begin{array}{l}\text { T. convencional } \\
(n=15)\end{array}$ & $\begin{array}{l}\text { T. vendajeneuromuscular } \\
\text { propioceptivo }(n=15)\end{array}$ & \\
\hline \multicolumn{4}{|l|}{ Antes } \\
\hline Media \pm DS & $7,93 \pm 1,43$ & $7,73 \pm 2,12$ & $p=0,000$ \\
\hline \multicolumn{4}{|l|}{ Después } \\
\hline Media \pm DS & $3,07 \pm 1,53$ & $6,13 \pm 1,12$ & \\
\hline
\end{tabular}

En la tabla 2 se observa que al aplicar el vendaje neuromuscular propioceptivo (VNP) existe una mejora del índice de discapacidad cervical en los pacientes que padecen dolor cervical crónico, de acuerdo a la prueba paramétrica t Student para muestras independientes. Se presenta una diferencia estadísticamente significativa $(p=0,000)$. Además, en el grupo experimental los pacientes obtuvieron mejores resultados en sus puntajes antes de la aplicación del VNP $(35,66 \pm 3,19)$, así como después de la aplicación del VNP $(29,8 \pm 3,09)$. 
Tabla 2. Efectividad del vendaje neuromuscular propioceptivo (VNP) y disminución del índice de discapacidad cervical en pacientes que padecen dolor cervical crónico atendidos en un hospital estatal. Lima, Perú

\begin{tabular}{|c|c|c|c|}
\hline & Grupo control & Grupo experimental & $\mathrm{t}$ Student \\
\hline & $\begin{array}{l}\text { T. convencional } \\
(n=15)\end{array}$ & $\begin{array}{l}\text { T. vendaje de neuromuscular } \\
\text { propioceptivo }(n=15)\end{array}$ & \\
\hline \multicolumn{4}{|l|}{ Antes } \\
\hline Media \pm DS & $34,3 \pm 1,43$ & $35,66 \pm 3,19$ & $p=0,000$ \\
\hline \multicolumn{4}{|l|}{ Después } \\
\hline Media \pm DS & $16,23 \pm 5,61$ & $29,8 \pm 3,09$ & \\
\hline
\end{tabular}

\section{DISCUSIÓN}

El dolor cervical es una lesión musculoesquelética que puede llevar a la discapacidad en el individuo. Es causado por múltiples factores y se puede presentar a cualquier edad. En la actualidad, ha aumentado el número de casos en edades tempranas (niños y adolescentes) ${ }^{(16,17)}$.

El dolor se localiza en la zona posterior o posterolateral de la región cervical (cuello) y se manifiesta de forma gradual. Los síntomas clínicos incluyen dolor y rigidez en el cuello, cefalea, mareos, y dolor que se propaga a los hombros y a las extremidades superiores. Los episodios recurrentes son comunes ${ }^{(18)}$.

La cervicalgia tiene, mayormente, un origen mecánico, es ocasionada por posturas mantenidas de cabeza, brazos, o ambos, por sostener o cargar pesos de manera estática, realizar movimientos repetitivos de la columna cervical o los miembros superiores, y también por no realizar pausas o descansos en el trabajo. Además, si la alteración mecánica es constante y repetida, se observa daño de los discos intervertebrales y hasta de las propias vértebras, lo que ocasiona una lesión nerviosa. El envejecimiento, las enfermedades reumáticas y los traumatismos dan lugar a una irritación de los nervios ${ }^{(19,20)}$.

La cervicalgia mecánica tiene un impacto en el estado funcional del paciente, interfiere en las actividades básicas tales como el sueño o el cuidado personal, así como en las actividades laborales y es, también, una causa común de discapacidad y absentismo laboral (4).

El VNP es conocido como una técnica novedosa y popular utilizada en la actualidad en múltiples tratamientos en los diferentes campos de las ciencias de la salud, la fisioterapia y en el deporte, y que se emplea para prevenir y rehabilitar lesiones, etc. Esta técnica supera en gran medida a los vendajes tradicionales (vendas elásticas y rígidas), ya que actúa sobre cinco sistemas fisiológicos: fascia, piel, músculo, articulaciones y sistemas circulatorio y linfático ${ }^{(19,20)}$.

El efecto del VNP fue más eficaz que el del tratamiento convencional. Así mismo, influyó en la mejora y en la disminución del dolor cervical mecánico. En nuestro estudio, se ha podido evidenciar la efectividad del VNP en este tipo de pacientes, al igual que en las investigaciones de Ay (21), Antúnez Sánchez ${ }^{(22)}$, Puerma-Castillo ${ }^{(23)}$ y Azatcam ${ }^{(24)}$.

En cuanto a la disminución del dolor, la aplicación del VNP ha tenido un mejor resultado como medio de tratamiento no invasivo al dolor cervical crónico, como también reportan El-Abd (25) y Öztürk ${ }^{(26)}$. La estimulación de los receptores nerviosos de la piel a través del VNP produce señales de tacto y presión, en donde las fibras alfa y beta crean un bloqueo en la transmisión del impulso doloroso; de esta manera, se proporciona información mucho antes de la llegada de las vías del dolor. Por lo tanto, el efecto analgésico se da porque las fibras alfa y beta, que son estimuladas por el vendaje neuromuscular propioceptivo y que son de conducción rápida, llegan primero a los centros nerviosos espinales y supraespinales, lo que impide que otras sensaciones, en este caso las dolorosas, sean percibidas ${ }^{(14)}$.

Por otro lado, al aplicar el VPN se ha evidenciado un efecto estadísticamente significativo en cuanto a cambios en la limitación funcional del paciente, hallazgo del que también informan Copurgensli (27), Saavedra-Hernández ${ }^{(28)}$, Saavedra-Hernández ${ }^{(29)}$ y Kavlak ${ }^{(30)}$. La limitación funcional es un problema causado por el dolor cervical, por lo que es necesario conocer los factores que influyen sobre la capacidad funcional ya que una intervención temprana 
evitaría la progresión de la discapacidad, mejoraría el estado de salud y la calidad de vida, con el desarrollo de estrategias para mitigar este problema ${ }^{(31)}$.

El estudio contó con los recursos humanos y materiales necesarios, con la disponibilidad de los pacientes del nosocomio, y se consideraron los principios bioéticos en investigación. Las limitaciones del estudio fueron el cálculo de la muestra no probabilística, que no permite generalizar los resultados; además, no se realizó seguimiento a los participantes, lo que se puede recomendar para próximos estudios.

Sobre la base de los resultados, se concluye que el grupo al que se le aplicó el VNP, tuvo un resultado más eficaz, en comparación con el grupo que recibió el tratamiento convencional: se evidencio que el vendaje neuromuscular propioceptivo influye en la mejora y en la disminución del dolor cervical mecánico y en la disminución del índice de discapacidad cervical.

Por último, se debe tener en consideración la aplicación del vendaje neuromuscular propioceptivo en pacientes con dolor cervical mecánico en los diferentes nosocomios públicos y privados como parte de la intervención fisioterapéutica.

Contribuciones de los autores: VDAT, JLF y CSB han participado en la concepción y diseño del estudio, recolección de datos, obtención de resultados, análisis e interpretación de los datos, redacción del informe, revisión crítica del manuscrito, asesoría estadística, técnica o administrativa, y aprobación de versión final informe.

Fuentes de financiamiento: Este artículo ha sido financiado por los autores.

Conflictos de interés: Los autores declaran no tener ningún conflicto de interés.

\section{REFERENCIAS BIBLIOGRÁFICAS}

1. Ariza Mateos MJ, Rodríguez Torres J, Negrín Ventura S, Cabrera Martos I, López López L, Valenza MC. Relación entre el perfil clínico y la tensión neural en personas con dolor cervical crónico. Rev Fisioterapia. 2018; 40(4): 192-8.

2. Gross A, Kay TM, Paquin JP, Blanchette S, Lalonde P, Christie T, et al. Exercises for mechanical neck disorders. Cochrane Database Syst Rev. 2015.

3. Käärï S, Laaksonen M, Rahkonen O, Lahelma E, Leino-Arjas P. Risk factors of chronic neck pain: a prospective study among middleaged employees. Eur J Pain. 2012; 16(6): 911-20.

4. Selva F. El vendaje neuromuscular. Art Divulgatio. 2008; 4(1): 39.

5. Tas $S$, Korkusuz F, Erden Z. Neck muscle stiffness in participants with and without chronic neck pain: a shear-wave elastography study. J Manipulative Physiol Ther. 2018. 41(7): 580-8.
6. Pasinato F, Bordin J, Santos-Couto-Paz CC, Alves Souza J, Corrêa ECR. Cervical-scapular muscles strength and severity of temporomandibular disorder in women with mechanical neck pain. Fisioter Mov. 2016; 29(2): 269-78.

7. Saavedra Hernández M. Fisioterapia en la cervicalgia crónica, manipulación vertebral y kinesiotaping [Tesis]. Granada: Universidad de Granada. Facultad de Ciencias de la Salud; 2012.

8. Hidalgo Medina AA. Prevalencia de la cervicalgia mecánica en los choferes profesionales de la cooperativa de transporte Vía Flores [Tesis]. Ambato: Universidad Técnica de Ambato. Facultad de Ciencias de la Salud; 2018.

9. Grimby-Ekman A, Andersson EM, Hagberg M. Analyzing musculoskeletal neck pain, measured as present pain and periods of pain, with three different regression models: a cohort study. BMC Musculoskeletal Disord. 2009; 10: 73.

10. Gustafsson E, Thomée S, Grimby-Ekman A, Hagberg M. Texting on mobile phones and musculoskeletal disorders in young adults: a five-year cohort study. Appl Ergon. 2017; 58: 208-14.

11. Stenberg G, Lundquist A, Fjellman-Wiklund A, Ahlgren C. Patterns of reported problems in women and men with back and neck pain: similarities and differences. J Rehabil Med. 2014; 46(7): 668-75.

12. Beaulieu L, Valenzuela C. Evaluación y manejo del dolor cervical facetario. Rev Med Clin Condes. 2014; 25(5): 728-31.

13. Cerezo-Téllez E, Torres-Lacomba M, Mayoral-Del-Moral O, Pacheco-da-Costa S, Prieto-Merino D, Sánchez-Sánchez B. Health related quality of life improvement in chronic non-specific neck pain: secondary analysis from a single blinded, randomized clinical trial. Health Qual Life Outcomes. 2018; 16(1): 207.

14. Villota Chicaíza XM. Vendaje neuromuscular: efectos neurofisiológicos y el papel de las fascias. Rev Cienc Salud. 2014; 12(2): 253-69.

15. Calero Saa PA, Cañón Martínez GA. Efectos del vendaje neuromuscular: una revisión bibliográfica. Rev Cienc Salud. 2012; 10(2): 273-84.

16. Zhou L, Huang YY, Chen DY, Zhang D, Luo QS, Wang Y, et al. Correlation between both neck/shoulder and low back pain and daily behavioral habits among middle school students in Shenzhen. 2018; 39(4): 469-73.

17. Fares J, Fares MY, Fares Y. Musculoskeletal neck pain in children and adolescents: risk factors and complications. Surg Neurol Int. 2017; 8: 72.

18. Hsu YH, Chen WY, Lin H, Wang WT, Shih YF. The effects of taping on scapular kinematics and muscle performance in baseball players with shoulder impingement syndrome. J Electromyogr Kinesiol. 2009; 19(6): 1092-9.

19. Kernozek T, Durall CJ, Friske A, Mussallem M. Ankle bracing, plantar-flexion angle, and ankle muscle latencies during inversion stress in healthy participants. J Athl Train. 2008; 43(1): 37-43.

20. Lim C, Park Y, Bae Y. The effect of the kinesio taping and spiral taping on menstrual pain and premenstrual syndrome. J Phys Ther Sci. 2013; 25(7): 761-4.

21. Ay S, Konak HE, Evcik D, Kibar S. The effectiveness of Kinesio Taping on pain and disability in cervical myofascial pain syndrome. Rev Bras Reumatol Engl Ed. 2017; 57(2): 93-9.

22. Antúnez Sánchez LG, De la Casa Almeid M, Rebollo Roldán J, Ramírez Manzano A, Martín Valero R, Suárez Serrano C. Eficacia ante el dolor y la discapacidad cervical de un programa de fisioterapia individual frente a uno colectivo en la cervicalgia mecánica aguda y subaguda. Aten Primaria. 2017; 49(7): 417-25.

23. Puerma-Castillo MC, García-Ríos MC, Pérez-Gómez ME, AguilarFerrándiz ME, Peralta-Ramírez Ml. Effectiveness of kinesio taping in addition to conventional rehabilitation treatment on pain, cervical range of motion and quality of life in patients with neck 
pain: a randomized controlled trial. J Back Musculoskelet Rehabil. 2018; 31(3): 453-64.

24. Azatcam G, Atalay NS, Akkaya N, Sahin F, Aksoy S, Zincir O, et al. Comparison of effectiveness of transcutaneous electrical nerve stimulation and kinesio taping added to exercises in patients with myofascial pain syndrome. J Back Musculoskelet Rehabil. 2017; 30(2): 291-8.

25. El-Abd AM, Ibrahim AR, El-Hafez HM. Efficacy of kinesio taping versus postural correction exercises on pain intensity and axioscapular muscles activation in mechanical neck dysfunction: a randomized blinded clinical trial. J Sports Med Phys Fitness. 2017; 57(10): 1311-7.

26. Öztürk G, Külcü DG, Mesci N, Silte AD, Aydog E. Efficacy of kinesio tape application on pain and muscle strength in patients with myofascial pain syndrome: a placebo-controlled trial. J Phys Ther Sci. 2016; 28(4): 1074-9.

27. Copurgensli C, Gur G, Tunay VB. A comparison of the effects of Mulligan's mobilization and kinesio taping on pain, range of motion, muscle strength, and neck disability in patients with cervical spondylosis: a randomized controlled study1. J Back Musculoskelet Rehabil. 2017; 30(1): 51-62.

28. Saavedra-Hernández $M$, Castro-Sánchez AM, Arroyo-Morales $M$, Cleland JA, Lara-Palomo IC, Fernández-de-Las-Peñas C. Short-term effects of kinesio taping versus cervical thrust manipulation in patients with mechanical neck pain: a randomized clinical trial. J Orthop Sports Phys Ther. 2012; 42(8): 724-30.

29. Saavedra-Hernández M, Arroyo-Morales M, Cantarero-Villanueva I, Fernández-Lao C, Castro-Sánchez AM, Puentedura EJ, et al. Shortterm effects of spinal thrust joint manipulation in patients with chronic neck pain: a randomized clinical trial. Clin Rehabil. 2013; 27(6): 504-12.

30. Kavlak B, Bakar Y. Investigation of the efficacy of different physiotherapy methods for neck pain. J Musculoskeletal Pain. 2012; 20(4): 284-91.
31. Marengo MF, Schneeberger EE, Gagliardi S, Maldonado Cocco JA, Citera G. Determinantes de discapacidad funcional en pacientes con espondilitis anquilosante en Argentina. Rev Argent Reumatol. 2009; 20(3): 20-5.

\section{Correspondencia:}

Cristhian Santiago Bazán

Dirección: Jr. Tiahuanaco 1493 Urb. Lima, Perú.

Teléfono: 941703104

Correo electrónico: cristhiansantiagob@gmail.com

\section{Recibido: 07 de marzo de 2019} Evaluado: 24 de junio de 2019 Aprobado: 03 de julio de 2019

(C) La revista. Publicado por Universidad de San Martín de Porres, Perú. (cc) ву Licencia de Creative Commons Artículo en acceso abierto bajo términos de Licencia Creative Commons Atribución 4.0 Internacional. (http://creativecommons.org/licenses/by/4.0/)

\section{ORCID iDs}

Cristhian Santiago Bazán (D) https://orcid.org/0000-0001-9073-4785

Violeta Del Aguila Torres C https://orcid.org/0000-0002-4372-1559

Janelle Lermo Fajardo (C) https://orcid.org/0000-0002-2992-7526 